\title{
Aspectos Psicosociales Antes y Después de la Competición del Comportamiento Agonista en Baloncesto
}

\author{
Psychosocial Aspects Before and After of the Competition of Agonist Behavior in Basketball
}

\author{
López-Niño $A A^{1}$
}

\begin{abstract}
:
Sport is composed of varied and broad factors, not only consists of developing a technique, physical preparation, or a form of play, its structure is a complex system Martín y Lago (2005). This complexity is due to the fact that, from the sporting point of view, decisional motor gestures are made, within the intentional technical actions, Anguera, (2005), being necessary to consider the cognitive, affective and human values that permeate training and competitions, that beyond the preparation of athletes, the coach must form good people and socially productive. One of the fundamental elements in the sports preparation is to work the psychological aspect of the athletes, specifically in the young athletes, because by means of it, the mental strength, the combativeness, the spirit of transcending are reflected, and thus the player he becomes an agonist, he does not stop fighting no matter how inexperienced he may be, looking for new challenges, supporting and managing better the triumphs and defeats that are inherent in the practice of sports. The study was conducted to determine the psychosocial aspects of the agonist behavior of the 16 basketball players of the youth category 2011 - 2012 of the men's team, of the School of Sports Sciences of the Universidad Anáhuac México, through the use of two instruments of measurement: competitive behavior and inventory of psychological performance of measurement; under the design of pretest - posttest, in a period from January 11 to June 10, 2017.
\end{abstract}

Keywords:

Psychosocial aspects, Agonist behavior, Basketball

\section{Resumen:}

El deporte está compuesto por factores variados y amplios, no solo consiste en desarrollar una técnica, preparación física, o una forma de juego, su estructura es un sistema complejo Martín y Lago (2005). Dicha complejidad se debe a que, desde el punto de vista deportivo, se realizan gestos motrices decisionales, dentro de las acciones técnicas intencionadas, Anguera, (2005), siendo necesario considerar los aspectos cognitivos, afectivos y valores humanos que permean entrenamientos y competiciones, que más allá de la preparación de los deportistas, el entrenador debe formar buenas personas y productivas socialmente. Uno de los elementos fundamentales en la preparación deportiva es el trabajar el aspecto psicológico de los atletas, específicamente en los deportistas jóvenes, porque por medio de ella, se refleja la fuerza mental, la combatividad, el ánimo de trascender, y así el jugador se vuelve agonista, no deja de luchar por muy inexperto que este sea, buscando nuevos desafíos, soportando y manejando mejor los triunfos y derrotas que son inherentes de la práctica deportiva. El estudio se realizó para determinar los aspectos psicosociales del comportamiento agonista, de los 16 jugadores de baloncesto de la categoría juvenil 2011 - 2012 del equipo varonil, de la Escuela de Ciencias del Deporte de la Universidad Anáhuac México, por medio del empleo de dos instrumentos de medición: conducta competitiva e inventario de rendimiento psicológico de medición; bajo el diseño de pretest - postest, en un periodo del 11 de enero al 10 junio de 2017.

Palabras Clave:

Aspectos psicosociales, Comportamiento Agonista, Baloncesto

Los aspectos psicosociales son las relaciones que surge de la interacción de dos factores definidos, uno compuesto por el trabajo, el medio ambiente y de las condiciones de organización, y el otro por, las capacidades del trabajador, sus necesidades, su cultura y su situación personal fuera del trabajo, todo lo cual, a través de percepciones y experiencias, pueden influir en la salud, el rendimiento y la satisfacción laboral (Consejería de Educación y Cultura, 2004).

En el campo deportivo y específicamente en el baloncesto, los aspectos psicosociales hace referencia a 
las condiciones presentes en entrenamientos, que hasta cierto punto están controladas, y las competiciones, que durante su desarrollo tiene múltiples variaciones que son manejadas según la pericia del jugador, porque debe tener presente a sus compañeros, adversarios, las situaciones que surgen durante las acciones de juego, las tareas que debe efectuar, además siendo afectadas por el contexto social que envuelve al partido, como son, la importancia que tiene el mismo, el ambiente formado por los asistentes y el rival en turno; esto afecta su rendimiento de manera positiva, negativa o neutra en los componentes motrices, afectivos, cognitivos y sociales; de esta forma, los factores psicosociales influyen en el espíritu de competición de cada jugador provocando que el rendimiento de cada uno de ellos sea distinto, pero tratando de tener una regularidad de forma conjunta. Para dimensionar el estudio es necesario revisar los aspectos psicosociales que se proponen por medio de los instrumentos de evaluación que están presentes antes, durante y después de la competición deportiva, (Mora \& Chapado, 2008; Especialistas Deportivos Asociados, 1995).

El espíritu de competición se viene dando desde tiempos de la Grecia antigua, siendo una de las bases de la sociedad helenística, en donde se competía por la superioridad en cualquier ámbito como podía ser el político, militar o incluso en la música; cuando una ciudad griega hacía un templo, otras intentarían hacer un templo mayor, esta especie de enfrentamiento en el que se buscaban el honor y la gloria, esta competición, es lo que se conoce como el Agón, y esta temática ha sido representada constantemente en el arte y literatura griega (López, 2016).

El Agón forma parte esencial de la civilización griega, este término hace referencia a la lucha en la que se busca obtener la victoria sobre un adversario digno de uno, para probar el valor y alcanzar la gloria, respetando ciertas reglas admitidas por ambos contendientes. El espíritu agonista es el ánimo de la competición, de la emulación, es el deseo de ser el primero, en pocas palabras ser el mejor (Samaniego,2011); las raíces del Agón están representadas en las obras de Homero, que describen la búsqueda del hombre por ser el mejor, esto queda representado por Aquiles. Su combate individual contra Héctor es un gran ejemplo de una lucha a muerte por la gloria y la areté, Gagliardi (2011). En cuyo caso, el objetivo de la lucha uno a uno hasta la muerte, la esencia más leal de oposición en la competencia era exhibir la virtud, el areté. El héroe luchaba por sí mismo y por el honor y la gloria, eligiendo morir en combate antes que vivir una larga y pacífica existencia, debido a dos conceptos clave: la inmortalización de su nombre junto con sus grandes hazañas, y el ideal de morir siendo joven y bello rehusando envejecer y volverse débil (Arena, 2012). A los nobles se les educaba en la búsqueda de la excelencia mostrada mediante la destreza guerrera y el valor heroico. Esta superioridad está íntimamente relacionada con el honor, su afán de distinción tiene su reflejo en la estimación y en la alta opinión que merecía a sus semejantes.

Con la llegada de democracia, el espíritu agonista se extenderá por todos los aspectos de la civilización griega, cuya manifestación más brillante son los innumerables concursos deportivos, artísticos e hípicos (Samaniego,2011); pero el Agón no es sólo el concurso que, opone a los diversos elementos de un mismo grupo, es también el proceso de oposición entre acusado y al acusador en un juicio, es la lucha verbal que opone a los dos personajes principales de una tragedia*, el debate en que se enfrentan ante la asamblea dos hombres y dos políticas, las luchas de prestigio entre las ciudades, el combate en que el ejército de hoplitas trata no de destruir al otro, sino de arrojarle fuera del campo de batalla.

En nuestros días somos herederos de espíritu por mejorar, el deseo de competir, el respeto por el rival, el afán de superación, la lucha por conocer y la perseverancia ante las adversidades de la vida, que se ve reflejado en los deportes a través del comportamiento agonista.

\section{Planteamiento del problema}

Dentro de las observaciones empíricas que el autor del presente estudio ha realizado en el Proceso de Preparación Deportiva de los deportes de equipo, específicamente en el baloncesto, se tiene una constante: los entrenadores no cubre las premisas del PPD, dedicándose primordialmente al componente técnico, seguido del táctico, que solo abordan por medio del sistema de juego que utilizan, y en algunos casos contados atienden el aspecto físico; esto se agrava cuando se trata de la iniciación y posterior especialización deportiva; donde los practicantes infantiles y jóvenes carecen del aspecto mental necesario para poder incrementar sus posibilidades de comprensión del juego y posterior elevación de su rendimiento; si hay que señalar un responsable directo de esta situación son los entrenadores a cargo, que no invierten tiempo a la preparación psicológica, esto se agrava cuando desconocen los factores propios de la psicología del deporte, y por ende sus deportistas en muchas ocasiones no alcanzan la victoria. Esta situación sirvió de motivo para determinar el comportamiento agonista del club juvenil de baloncesto varonil "Leones Anáhuac" de la Escuela de Ciencias del Deporte (ECD) de la Universidad Anáhuac México, ubicada en el campus norte; donde se explicaron los aspectos psicosociales que median en la lucha competitiva, antes y después del PPD, el investigador no intervino en la conducción de entrenamientos y/o partidos de competencia.

Con los resultados obtenidos, se pretende elaborar un plan psicológico de intervención para ayuda y mejora de los aspectos psicosociales abordados por medio de los test empleados.

\footnotetext{
* Precisamente de la palabra Agón proceden las palabras protagonista y antagonista
} 


\section{Antecedentes}

La competición hace referencia a un tipo de enfrentamiento que se limita en la mayoría de los casos a lo deportivo y que supone el respetar determinado tipo de reglas o reglamento, cumplir con determinados requisitos y tener en vista la obtención de un buen resultado competitivo, originando que "el contacto directo con el adversario, así como la atmósfera creada por los espectadores, el dominio del campo de juego y la importancia del mismo, influyan fuertemente en el ánimo del jugador" (Navlet, 2011); por lo que el contexto en que se desenvuelve el jugador durante la competición deportiva afecta la toma de decisiones y la combatividad, de forma positiva, negativa o neutra, según la personalidad, experiencia, grado de entrenamiento y nivel de eficiencia; originando en el deportista el impulso de superación, que esta está íntimamente ligado a la sobrevivencia y a las tendencias de dominio que posee el hombre.

Para Garzarelli (2015) "este impulso se presenta muy tempranamente en nuestra vida y es posible observarlo con claridad en los juegos infantiles; en estos, el niño repite en forma activa aquello que ha vivenciado previamente en forma pasiva". Según Trenchi (2016) "el juego sirve además como conducta que modela, limita, y recrea la fantasía del niño". Dado que existe una profunda relación que se puede establecer entre el juego y el deporte, ambos tienen aspectos similares con el común denominador del placer como afecto primordial.

Desde la infancia se "modelan carácter y personalidad, el temperamento juega un papel importante siendo innato, heredado e inmodificable; los primeros son altamente determinados por el núcleo familiar y las primeras instituciones, como la escuela, incluso la iglesia, a las que accede el niño" (Eraso, 2012); el club deportivo y/o la escuela de iniciación deportiva, también operan como modificador, contenedor y canalizador del temperamento, carácter y personalidad infantil.

Los niños al competir desde los juegos lúdicos tradicionales y populares, pasando por los deportes que se adecuan a sus posibilidades, van paulatinamente "desarrollando habilidades físicas, cognitivas y psicológicas con las que posteriormente podría manejarse con mayor facilidad y éxito en la vida adulta" (Rojas, Robinson, \& Viviescas, 2009).

En estos juegos encontramos implícita, la satisfacción imaginaria de necesidades vitales, dando sentido a toda la estructura personal física como la psicosocial. Estas necesidades vitales durarán toda la vida y posteriormente pueden ser satisfecha por las profesiones, el deporte y otras actividades (García, 2016).

Para Garzarelli (2015), en todas estas necesidades vitales "es posible la sublimación constitucional y natural de la agresividad humana, con el consecuente beneficio secundario". La sublimación es la posibilidad de que las pulsiones sexuales y agresivas, se manifiesten con toda su energía, sustituyendo un objetivo inaccesible por otro situado fuera de la sexualidad o la agresividad y valorado por la sociedad, como lo son: el arte, la cultura, la ciencia y en nuestro caso, el deporte; en esencia, pretende resolver el conflicto entre la necesidad, determinada por el objeto de satisfacer el principio del placer y la necesidad de cumplir con el principio de la realidad(Córdoba, 2012).

Una adecuada competencia infantil y juvenil favorece la evolución a diferentes, posteriores y estructurados estadios que incrementan y facilitan la madurez físico emocional del practicante; para lo cual la forma de enseñanza es trascendental en los deportes de equipo, denominados también de cooperación - oposición por Hernández, (2005); de aquí la importancia del juego deporte a edades tempranas. El niño, después el joven y posteriormente el adulto, al irse superando en sus metas y marcas, por iniciativa propia o por la conducción del profesor/entrenador, va adquiriendo un elemento profundo del desarrollo, mejora y perfeccionamiento sus propios recursos psicosociales personales.

\section{Propósito de la investigación}

Determinar los aspectos psicosociales del comportamiento agonista, antes y durante la competición, de los jugadores de baloncesto de la categoría juvenil 2011 - 2012 del equipo varonil "Leones Anáhuac", de la Escuela de Ciencias del Deporte de la Universidad Anáhuac México, por medio del empleo de dos instrumentos de medición.

\section{Fundamentos teóricos}

La conducta se refiere a la forma que un organismo se conduce en relación con los demás, según una norma moral, social o cultural; se hace referencia también, a la conducta global de un determinado grupo social en sus relaciones hacia los otros. En ocasiones se le emplea como sinónimo de comportamiento, pero es incorrecto hacerlo, pues la conducta implica una actividad consciente, observable y repetible.

El comportamiento es la forma de proceder que tienen las personas, en relación con su entorno y los estímulos que recibe del mismo, este puede ser consciente 0 inconsciente, voluntario o involuntario, público o privado, según sean las circunstancias que afecten al organismo (Reyes,2012). El hecho de que exista una similitud entre conducta y comportamiento lleva a tomarlos como sinónimos, cuando existe una pequeña brecha que los separa que es la contingencia, que en define como en dependencia de.

De esta forma, tenemos que el comportamiento agonista es un concepto más general al de la conducta agresiva pues incluye cualquier actuación de oposición a otros, como la competición, el enfrentamiento, la desobediencia y, por supuesto, la conducta agresiva; para Verísimo (2012), "la conducta agresiva en determinadas circunstancias es adaptativa y, en cualquier caso, constituye un elemento normal del repertorio de conductas del ser humano" Para López (2016), el estado agonista es:

Todo impulso psicológico de luchar, toda la agresividad competitiva inconsciente, es un redireccionamiento de la agresividad, este estado se pone de manifiesto desde edades tempranas plasmado en la competencia que se busca o adquiere con otro ser humano, buscamos el salir 
victoriosos de diversas circunstancias dadas; al lograrlo se va produciendo o fraguando una satisfacción o placer que pudieran conllevar a un sentido de exigencia natural, en el afán de ir en busca de una victoria y de lucha, encontrando satisfacción en ello, siendo dependiente de la personalidad de cada individuo.

Para reforzar la cita anterior, Aldana (2013), señala que "la agresión es un instinto innato, y al ser un instinto se vuelve necesaria para la supervivencia del hombre y para el desarrollo integral de la persona", por el contrario, Moise (2011) manifiesta que "la violenta es una conducta aprendida socialmente, que nace en el seno familiar y se cristaliza en las escuelas"

A mediados del siglo XX, la conducta agresiva como tema central, inicio a ser estudiada por la psicología, sociología, biología y la etología, Chapi (2012), al ser un objeto de estudio multidisciplinario, ha sido abordado desde otras disciplinas como lo son las actividades físicas y deportivas.

El deporte es parte de la sociedad que nos permite a través de su practica el inculcar valores y actitudes para la vida en donde los niños y jóvenes puedan irse conformando una determinada manera de ser, hay condiciones psicológicas que se trasmiten de una manera común en los diferentes deportes como la disciplina, la responsabilidad, la toma de decisiones, la proactividad y la concentración, por mencionar algunas, siendo de los más buscados el fomentar, desarrollar y consolidar el comportamiento agonístico, llamado coloquialmente en el deporte: espíritu de lucha, combatividad o competitividad (Dorado, 2012).

El deporte está compuesto por diversos factores variados y amplios, no solo consiste en desarrollar una técnica, preparación física, o si fuera el caso, un sistema de ataque y defensa, siendo "su estructura de juego deportivo un sistema complejo" Martín y Lago (2005) de actividad. Dicha complejidad se debe a que, desde el punto de vista deportivo, "se realizan gestos motrices decisionales, dentro de las tareas motrices técnicas intencionadas (acciones procedimentales), con el objetivo de captar la realidad", Anguera (2005), siendo necesario considerar los aspectos cognitivos y afectivos, y los valores humanos que deben permear entrenamientos y competiciones, más allá de la preparación de los deportistas, el entrenador debe formar buenas personas y productivas socialmente. Para la psicóloga cognitiva conceptual, Susana Hernández, directora de HDZ Consultora, mencionada por Sarasqueta

(2014), señala que:

El deporte es un vehículo no sólo para entrenar distintas habilidades físicas sino también para entrenar diferentes competencias o habilidades humanas, como lo son las relaciones sociales, el compañerismo, la solidaridad, el trabajo en equipo, la disciplina, entre otras. En ese sentido, la práctica de un deporte moldea determinados rasgos de la personalidad. En un estado de generación de máxima adrenalina y aumento de la ansiedad, propia de una competición, se pueden alterar ciertas cualidades de la persona, como la manifestación de la ira, el miedo o la autoconfianza, que no se sabía que se tenía.

Dado que a través del deporte se fomentan conceptos de acción para la vida, y estando inmersos en una sociedad en la cual el desempeño por competencias va marcando el cambio en sistema social actual; según Bustamante (2008), es necesario desarrollar personas combativas que tengan la determinación de realizar acciones trascendentales estando en una lucha y superación personal constante.

Los hermanos Carrasco (2014), establecen que "la agresividad en todos los seres y en el hombre no se puede suprimir, la negación de la misma puede causar perturbaciones en el equilibrio psíquico, no está claro que la agresividad sea destructiva". Las actividades físicas y deportivas se muestran como una de las pocas acciones humanas en que la pulsión agresiva puede ser liberada completamente.

Según Cagigal (1990) "el deporte es la gran ocasión de elaborar la agresividad de forma positiva, la sociedad recurre a sistemas o formas capaces de defenderla del excedente agresivo de los hombres, canalizando la agresión a formas aceptables"

En palabras de Piqueras (2012):

Una de las características que los deportistas deben desarrollar es la denominada combatividad, no es otra cosa que la capacidad que tiene un deportista para hacer todo lo necesario con el fin de alcanzar sus objetivos. Esta particularidad ha recibido otro tipo de denominaciones, como: determinación, competitividad, persistencia, compromiso, perseverancia, etc

La competitividad o combatividad, como componente de la fortaleza mental, es un requisito necesario e importante en cualquier tipo de actividad físico-deportiva, sin ella no es posible hablar de un deportista triunfador, en toda la extensión de la palabra, a pesar de su capacidad físico técnica elevada, será si acaso, un jugador promedio, con destellos de acciones motrices de alta calidad de ejecución, pero que no logrará trascender. El comportamiento agonista, según Neri (2005), "es uno de los aspectos fundamentales a trabajar en la preparación psicológica de todo deportista en general, siendo uno de los aspectos más difíciles de conseguir y consolidar". Un deportista con menores capacidades físicas, técnicas y tácticas, con una correcta y mayor preparación y el desarrollo de sus habilidades psicológicas, que su oponente, le puede permitir acceder a la victoria. Los factores que constituyen el aspecto agonista, según Sanabria (1985), son:

- $\quad$ Agresividad, "es un factor del comportamiento normal puesto en acción para responder a necesidades vitales, que protegen la supervivencia de la persona y de la especie, sin que sea necesaria la destrucción del adversario", Guerri (2012).

- $\quad$ Tensión, "es la respuesta del organismo ante la presión física, cognitiva, emocional que se experimentan por diversas causas" (Piqueras, Ramos, Martínez, \& Oblitas,2009).

- $\quad$ Sobrecompensación, "refleja un impulso potente de lograr un margen extraordinario de desarrollo, frecuentemente más allá de un rango normal. Puede tratarse de pasos útiles para logros excepcionales o inútiles de excesivo perfeccionismo" (Ruiz, 2013).

- $\quad$ Exhibicionismo, "se refiere a la necesidad y al patrón de conducta impulsiva para mostrar a otras personas partes desnudas del cuerpo o conductas sexuales y eróticas" (Pérez, Borras, \& Zubieta, 2007). 
- $\quad$ Motivación, "se trata de una jerarquía de factores que incentivan a las personas para satisfacer sus necesidades; a medida que se satisfacen surgen otras que cambian o modifican el comportamiento de la persona" (Quintero,2007).

Vives (2012), establece que:

Las habilidades psicológicas como la toma de decisiones, el comportamiento agonístico, la combatividad, están presentes en cualquier actividad y situación, desde la más cotidiana a la más extraordinaria, en cualquier ámbito de la vida. Las personas tenemos recursos diferentes y formas de afrontar las situaciones que pueden ser más o menos adecuadas ante las exigencias de la situación. Con frecuencia los recursos propios no son suficientes, o bien el problema o el objetivo a lograr es demasiado complejo.

Para Vasalo (2001), la competitividad o combatividad configura deportistas que tienden a buscar desafíos, que se esfuerzan por conseguir sus objetivos y que persisten en su actividad a pesar de derrotas o lesiones, obteniendo placer por la actividad que realizan.

La práctica deportiva es una forma de educación, que, al igual, de la que se recibe en el aula, ambas son un proceso social, teniendo como fundamento primario mantener y consolidar los procesos de formación integral de los participantes.

\section{Método}

El tipo de investigación es explicativa, esta clase de estudios pretende buscar y encontrar las razones $y / 0$ causas que provocan ciertos fenómenos, bajo el un enfoque cuantitativo con diseño transversal Pretest Postest; donde se emplearon dos instrumentos de medición, el primero de ellos es un Cuestionario de Conducta Competitiva de Mora y Chapado (2008), en cinco dimensiones, que a continuación se detallan, y que contienen un total de 50 ítems

-Preocupación y rendimiento (compuesto por 13 ítems, los cuales son los enumerado con el: $3,4,8,9,12,13$ 18, 24, 36, 37, 40, 41, 45). - La Preocupación es la acción vinculada a la generación de zozobra o nerviosismo, o a ocuparse con antelación de algo. El Rendimiento se refiere a la proporción que surge entre los medios empleados para obtener algo y el resultado que se consigue.

-Potencialidad de rendimiento (integrado por 9 ítems, siendo los reactivos número $1,5,7,22,25,28,44,47$, 48). - Se refiere a las capacidades que una persona puede desarrollar. Abarca incluso aquellas capacidades que no existen de manera natural en cada uno de nosotros pero que se pueden trabajar, por un medio adecuado, a partir del interés que nos producen.

-Concentración (9 ítems articulados en los números 2, 6 , $11,16,20,27,31,39,46)$. - Es el proceso de la mente que consiste en centrar voluntariamente la atención sobre un objetivo.

-Entrenamiento mental (agrupado en 11 ítems, los cuales son el 10, 17, 19, 21, 26, 32, 38, 42, 43, 49, 50). - Es la aplicación de ciertas habilidades psicológicas (actitud, comportamiento, comunicación, autorregulación, autoconfianza, autoconcepto, autoestima, toma de decisiones) con el fin de mejorar y perfeccionar el rendimiento deportivo.

-Motivación (compuesto por 8 ítems, siendo el 14, 15, 23, 29, 30, 33, 34, 35). - Expectativas de rendimiento, obstinación ante presión extrema y reto para actuar mejor, disfrute agonístico, gusto por ser estimulados, establecimiento de objetivos y metas insatisfacción ante niveles alcanzadas de actuación.

El segundo Test es el de Rendimiento Psicológico, fue elaborado por la empresa de Especialistas Deportivos Asociados (EDA), para su uso en el área de evaluación funcional y soluciones deportivas (1995), siendo coordinado por el Mtro. Víctor Hernández López, en siete dimensiones, que a continuación se detallan, y que contienen un total de 42 ítems

-Autoconfianza (son 6 ítems, de los cuales son el 1, 8, 15, 22, 29, 36). - Es el convencimiento íntimo de que uno es capaz de realizar con éxito una determinada tarea o misión, o bien elegir la mejor alternativa cuando se presenta un problema, es decir tomar la mejor decisión. Es confiar en que en general uno va a salir airoso de una situación, por difícil que parezca.

- Energía positiva y negativa (integrado por 12 ítems, siendo los 6 primeros para la parte negativa y son el 2, 9, 16, 23, 30, 37; y la parte positiva son el 6, 13, 20, 27, 34, 41). - La canalización de la energía de un deportista puede ser positiva o negativa y esto se medirá en función de las consecuencias obtenidas en las ejecuciones técnicas. Si el deportista está demasiado relajado para afrontar la competición se utilizan técnicas de energización; Si, por el contrario, el deportista está sobreexcitado o ansioso se utilizan técnicas de relajación. - Control de la atención (contado por 6 ítems y son el 3, $10,17,24,31,38)$. - Se trata de una variable decisiva para poder detectar e interpretar las demandas de cada situación, y que el control atencional correcto va a proporcionar una mejora en la toma de decisiones.

-Control visual y de imagen (conjuntado por 6 ítems y son el 4, 11, 18, 25, 32, 39). - La noción sobre el mundo se crea a partir de las representaciones mentales (imágenes, conceptos, esquemas, etc.), que almacenamos en la mente de las cosas y hechos que observamos a diario; es como si la realidad la dibujáramos en nuestra memoria, pero no de manera exacta, con imperfecciones, inexactitudes etc.

-Nivel de motivación (es agrupado en 6 ítems y son el 5, $12,19,26,33,40)$. - Se cual trata de una jerarquía de necesidades y factores que motivan a las personas; esta jerarquía identifica cinco categorías de necesidades y considera un orden jerárquico ascendente de acuerdo con su importancia para la supervivencia y la capacidad de motivación.

- Control de actitud (esta dimensión esta agrupada en 6 ítems y son el 7, 14, 21, 28, 35, 42). - Es el caso del deportista, la predisposición que va a determinar su comportamiento, siendo adquirida por medio del aprendizaje, mostrando un componente motivacional que no es observable directamente, siendo perdurable a través del tiempo como un elemento evaluador positivo, negativo o neutro que implica relación entre aspectos cognitivos, afectivos y de actuación 


\section{Muestra y selección de participantes}

El estudio se realizó dentro de la Universidad Anáhuac México Campus Norte, en la Escuela de Ciencias del Deporte y se centró en el equipo de baloncesto varonil del "Club Leones Anáhuac" de la categoría juvenil A, integrado por $\mathrm{N}=16$ jóvenes que nacieron entre 2001 y 2002 , que cursan el $2^{\circ}$ y $3^{\circ}$ grado de secundaria. Durante el período del 9 de enero al 12 de junio de 2017, teniendo entrenamientos los días lunes, miércoles y viernes, en un horario: 17:00 a 19:00 hrs. Durante el periodo descrito los jóvenes acumularon un total de 30 partidos (24 oficiales y 6 de preparación), teniendo un porcentaje de efectividad de partidos ganados y perdidos 95\% (38-2); cubriendo un total de 120 horas de entrenamiento cubiertas en 60 sesiones.

\section{Procedimiento}

Durante el periodo señalado se aplicó dos veces el Test de Conducta Competitiva y el Inventario de Rendimiento Psicológico, el pretest se llevó a cabo los días 11 y 13 de enero de 2017; el postest se realizó los días 8 y 10 de junio de 2017; durante el tiempo señalado hubo 60 entrenamientos y 30 competencias; de los cuales se observaron de forma directa 20 entrenamientos, uno cada semana, específicamente los días miércoles y se asistió a 15 partidos, 2 de preparación y 13 de competencia; aquí señalamos que no te intervino de ninguna forma en alguna de las actividades propias del proceso de preparación.

\section{Resultados}

La tabla 1 muestra los resultados de las cinco dimensiones del Test de Conducta Competitiva, que se aplicaron en los dos momentos señalados, siendo estos aspectos psicosociales, los que integran parte del comportamiento agonista, así tenemos los datos siguientes:

Tabla 1

Resultados de Conducta Competitiva.

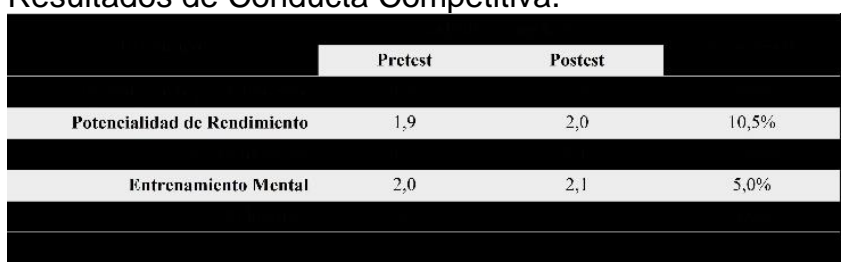

Es importante señalar que en todas las dimensiones hubo un incremento, excepto en lo relacionado con la motivación, este aspecto no se modificó. La figura 1 muestra la integración del test para determinar parte del comportamiento agonista, donde se muestra un pequeño incremento; que según el resultado de la prueba es de aproximadamente $7 \%$, donde el pretest alcanzo 1,95 y el postest 2,09.

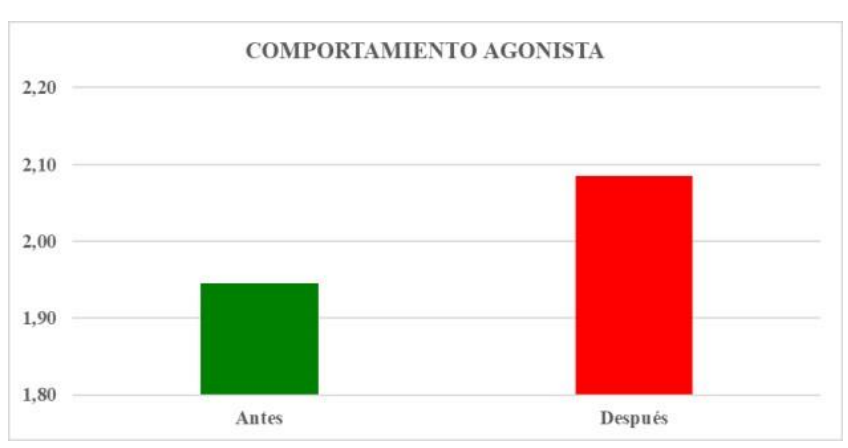

Figura 1. Mora \&Chapado, 2008

La tabla 2 muestra los resultados de las siete dimensiones del Test de Rendimiento Psicológico, que se utilizaron en las fechas mencionadas líneas arriba, donde estos aspectos psicosociales, integran junto con las dimensiones del test anterior el comportamiento agonista, así tenemos los datos siguientes:

Tabla 2

Resultados del Rendimiento Psicológico

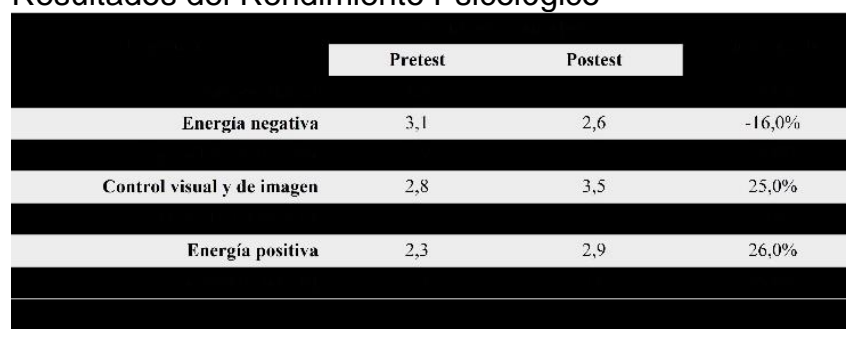

Como se puede observar los resultados de las pruebas señalan un incremento importante, debido a la acumulación de entrenamientos y el resultado de las competiciones; no existe otra razón debido a que el entrenador no contemplo un plan de intervención psicológica. Estos aspectos psicosociales y los del test anterior integran el comportamiento agonista de los jugadores el espíritu de lucha descrito en el Agón de los griegos implementado en las actividades físicas. Cabe destacar, que nuevamente el aspecto motivacional tiene niveles muy bajos, haciendo una referencia cruzada con el test de conducta competitiva en la dimensión señalada tenemos que corroborar que los integrantes del equipo posiblemente necesiten un plan de intervención en el aspecto mencionado. La figura 2 muestra la integración del Test para determinar el Rendimiento Psicológico Grupal, donde existe un incremento del 15;0\% en relación con los momentos de aplicación del cuestionario, siendo el primer resultado 2,8 y el final de 3,2. 


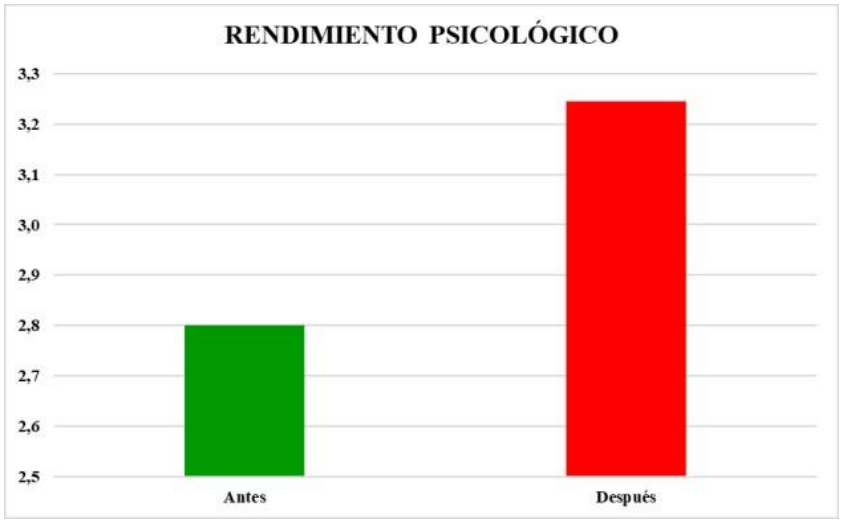

Figura 2 Especialistas Deportivos Asociados, 1995

\section{Conclusiones}

-Durante el periodo de estudio el equipo acumulo un total de 30 partidos (24 oficiales y 6 de preparación), teniendo un porcentaje de efectividad de partidos ganados y perdidos $93.3 \%$ (28-2); cubriendo un total de 120 horas de entrenamiento cubiertas en 60 sesiones.

-El equipo Leones Anáhuac, de forma general, sus jugadores han logrado una mayor control emocional y mejor manejo de la frustración; por lo que, la capacidad ante el esfuerzo ha crecido, provocando una disminución de la ansiedad, como lo señalan los resultados de la prueba psicológicas.

-El equipo ha desarrollado un estilo de juego agresivo, por medio de las defensas de presión y el rompimiento rápido, siendo penetrantes e incisivos; esto se ha logrado con una base física genérica, potencializado con el trabajo específico, a través del sistema de juego implementado.

-Desde el punto de vista psicopedagógico han logrado discriminar, visualizar e incrementar su concentración, esto quedó demostrado en la mejor toma de decisiones durante las competiciones en las que participaron.

- La constancia y continuidad de los entrenamiento y competiciones por si solos, incrementan el rendimiento del equipo de baloncesto, esto queda demostrado con el resultado de los test en los dos momentos que se aplicaron; es indudable que con un programa de intervención psicológica estas dimensiones se pueden potencializar.

- Una de las dimensiones que más nos llama la atención es la motivación (grupal), al no tener variación alguna. Una de las causas probables, se pueden encontrar en los niveles de la teoría de las necesidades (motivación), que establece que en cuanto están cubiertas estas en un gran porcentaje, se accede al siguiente nivel y la forma de motivación debe cambiar.

-Con respecto a esto último, los jugadores provienen de un estrato socioeconómico de clase media, donde sus necesidades básicas, de seguridad, de afecto y pertenencia, parece que están satisfecha; este grupo de jugadores está en la búsqueda de la estima propia (el reconocimiento hacia su persona, el logro particular y el respeto hacia los demás; al satisfacer estas necesidades, las personas tienden a sentirse seguras de sí misma y valiosas dentro de una sociedad).

- Con base a los datos arrojados por los test es indudable que el proceso de preparación deportiva, independientemente de que no se tenga un plan de intervención psicológica, señala incrementos en los aspectos psicosociales únicamente por el trabajo técnico - táctico - físico.

- Después de realizado el estudio surge la propuesta de elaborar un programa de intervención psicológica, para que sea aplicado por el entrenador.

\section{Referencias}

Aldana, R. (2013/12/26). Psicopedia. Agresividad: ¿nacemos violentos o aprendemos a serlo? Disponible de http://psicopedia.org/2335/agresion-nacemos-violentos-oaprendemosa-serlo/

Anguera, M. (2005). Planteamiento metodológico al servicio de la comprensión de la complejidad en los deportes de equipo. En Martín, R. \& Lago, C. Deportes de equipo: comprender la complejidad para elevar el rendimiento. pp. 133 - 148. Barcelona, España: INDE.

Arena, M. (2012). El espíritu competitivo griego: el Agón. Disponible en: http://www.pokexperto.net/foros/index.php?topic=28913.0; nowap

Bustamante, M. (2008). Método formación - acción en el desarrollo de competencias profesionales. Cuadernos de Docencia Universitaria, Versión electrónica 1 (1). Disponible de: http://dta.utalca.cl/ojs/index.php/cdocencia/article/viewFile/10/22 Cagigal, J. (1990). Deporte y agresión. Madrid, España: Alianza.

Carrasco, D., Carrasco, D. y Carrasco, D. (2014). Psicología del deporte. Universidad Politécnica de Madrid. Disponible en: http://futbolcarrasco.com/wpcontent/uploads/2014/08/futbolcarrascoin ef4curso4.pdf

Chapi, J. (2012). Una revisión psicológica a las teorías de la agresividad. Revista electrónica de psicología Iztacala, 15 (1). Disponible en: http://www.iztacala.unam.mx/carreras/psicologia/psiclin/vol15num1/V ol15No1Art5. pdf

Córdoba, R. (2012/04/03). Psicoletra. La sublimación. Disponible en: http://www.psicoletra.com/2012/04/la-sublimacion.html

Consejería de Educación y Cultura, (2004). Prevención de riesgos específicos en centros educativos. Disponible en: https://www.carm.es/web/pagina?IDCONTENIDO=3794\&IDTIPO=2 $46 \&$ RASTRO $=\mathrm{c} 801 \$ \mathrm{~m} 4331,4330$

Dorado, A. (2012). El fomento de la deportividad a través de la educación en valores y el juego limpio. Revista Andaluza de Documentación sobre el Deporte, $3 . \quad$ Disponible en: https://www.diba.cat/documents/467020/1973025/fomento+de+la+dep ortividad.pdf/851de6d4-2c9a-4aed-a72a-3a463944086d

Especialistas Deportivos Asociados (1995). Instrumentos y protocolos de psicodiagnóstico deportivo. CDMX, México: ENED CONADE

Eraso, I. (2012/06/04). Emocreativos. Diferencia entre temperamento, carácter y personalidad. Disponible en: https://emocreativos.com/2012/06/04/diferencia-entretemperamentocaracter-y-personalidad/

García, A. (2016/06/23). Sociólogos. Sobre la satisfacción de las necesidades humanas. Disponible en http://ssociologos.com/2016/06/23/la-satisfaccion-lasnecesidadeshumanas/

Gagliardi, G. (2011). La Ilíada de Homero, lectura y comentarios. Disponible de: http://letrasuruguay.espaciolatino.com/aaa/gagliardi_guillermo/la_iliad a_de_homero.htm 
Garzarelli, G. (2015). La competencia en el deporte. Psicología del deporte, [online] Sevilla, España: psicologia-online. Disponible en http://www.psicologiaonline.com/ebooks/deporte/competencia.shtml

Gould, D., Dieffenbach, K., \& Moffett, A. (2002). Psychological Talent and its development in olimpic champions. Journal of Applied Sport Psychology, 14, 177-210.

Guerri, M. (2012). La agresividad, conceptos y teorías. Psicoactiva. Disponible en: https://www.psicoactiva.com/blog/la-agresividad/

Hernández, J. (2005). Análisis de las estructuras del juego deportivo. Fundamentos del deporte. Barcelona, España: INDE.

Loo, V. (2011). Psicología infantil y de la adolescencia. Teoría psicológica. Sigmund Freud. Disponible en: http://www.maestroloo.com/maestroloo/psicologaclnica/teoriapsicoana liticasigmundfreud

López, M. (2016). Desarrollo de la combatividad a través de métodos intensivos en la preparación física del equipo jaguares categoría cadetes. (Documento inédito). CDMX, México: ENED

López, R. (2016). El Argón. El educador físico. Disponible en: https://ricardoglopez.blogspot.mx/2016/01/el-agon_14.html

Martín, R. \& Lago, C. (2005). Deportes de equipo: Comprender la complejidad para elevar el rendimiento. Barcelona, España: INDE

Moise, C. (2011). Psicoanálisis y comunidad. La violencia es una conducta socialmente aprendida. Disponible en http://psicoanalisisycomunidad.blogspot.mx/2011/09/laviolencia-esuna-conducta.html

Mora, J. \& Chapado, F. (2008). Visión actual de la psicología. Sevilla, España: Wanceulen.

Navlet, M. (2011). Ansiedad, Estrés y Estrategias de Afrontamiento en el ámbito deportivo: Un estudio centrado en la diferencia entre deportes. Universidad Complutense de Madrid Facultad De Psicología. España.

Pérez, M., Borras, J., \& Zubieta, X. (2007). Exhibicionismo. Sexo. Disponible en: http://www.elmundo.es/elmundo/2007/08/08/sexo/1186590377.html

Piqueras, J. (2012). Fortaleza Mental, Clave del rendimiento deportivo. ¿Qué se entiende por Fortaleza Mental? Disponible en http://www.fnclm.com/wordpress/wpcontent/uploads/Fortaleza_Menta 1.pdf

Piqueras, J., Ramos, V., Martínez, A., \& Oblitas, L. (2009). Emociones negativas y su impacto en la salud mental y física. Suma Psicológica, $\begin{array}{lllll}\text { Revista Digital } 16 & \text { (2). Disponible de: }\end{array}$ http://www.redalyc.org/pdf/1342/134213131007.pdf

Quintero, J. (2007). Abraham Maslow y su teoría de la motivación humana. Venezuela: Universidad Fermín Toro. Disponible de: http://doctorado.josequintero.net/

Reyes, R. (2012/12/8). Diferencia entre conducta y comportamiento. Psicología. Disponible en: http://comoporque.blogspot.mx/2012/04/diferencia-entre-conductay.html

Rojas, L. Robinson, I. \& Viviescas, M. (2009). El juego como potencializador del desarrollo del pensamiento lógico matemático, en niños de 5 a 6 años del grado transición, del Colegio Club de Desarrollo Mundo Delfín. Universidad de San Buenaventura: Bogotá, Colombia.

Ruiz, J. (2013). Las tres fases teóricas Adlerianas. Adleriana. Disponible en: http://adleriana.blogspot.mx/2013/03/conceptos-teoricosfundamentales-2_10.html

Samaniego, S. (2011). La civilización del Agón. La tortuga de Aquiles. Disponible en: https://asusanazan.wordpress.com/2011/02/13/lacivilizacion-del-agon/

Sanabria, M. A. (1985). Psicología del deporte y del ejercicio físico. Cúpula, Revista Digital, 9 (8). Disponible en: http://www.binasss.sa.cr/bibliotecas/bhp/cupula/v9n181985/art3.pdf
Sarasqueta, G. (2014). Deporte y personalidad. Disponible en: http://www.lavanguardia.com/estilos-devida/20140828/54414439029/deporte-y personalidad.html

Trenchi, N. (2016). Padres hoy. La competencia en los niños. Disponible en http://www.padreshoy.uy/la-competencia-los-ninos-n964220

Vasalo, C. (2001). Competitividad en Deportes Infantiles. PubliCE Standard, Revista Digital. Disponible en: https://gse.com/es/journals/publice-standard/artículos/competitividad-endeportes-infantiles-97

Verísimo, P. (2012). Agresión y violencia, una dimensión social. Clip Psicólogos online Disponible en https://clicpsicologos.wordpress.com/2012/04/18/agresion-yviolenciauna-dimension-social/

Vives, J. (2012). Psicología del rendimiento. ¿Qué es la psicología del rendimiento? Disponible https://joanvives.wordpress.com/2012/10/15/blog-personal-dejoanvives-ribo/

Weinberg, R. y Gould, D (2010). Fundamentos de psicología del deporte y del ejercicio físico. Madrid: Editorial Medica Panamericana. 